Cahiers $d u$ MONDE RUSSE

\section{Cahiers du monde russe}

Russie - Empire russe - Union soviétique et États indépendants

55/3-4 | 2014

Varia

\title{
Pierre Gonneau, Ecatherina RAI, éds. Écrire et réécrire l'histoire russe d'Ivan le Terrible à Vasilij Ključevskij (1547-1917)
}

\section{KORINE AMACHER}

\section{(2) OpenEdition}

1 Journals

\section{Édition électronique}

URL : http://journals.openedition.org/monderusse/8012

DOI : $10.4000 /$ monderusse. 8012

ISSN : $1777-5388$

Éditeur

Éditions de l'EHESS

\section{Édition imprimée}

Date de publication : 1 juillet 2014

Pagination : $327-332$

ISBN : 978-2-7132-2441-6

ISSN : $1252-6576$

\section{Référence électronique}

KORINE AMACHER, «Pierre Gonneau, Ecatherina RAI, éds. Écrire et réécrire l'histoire russe d'Ivan le Terrible à Vasilij Ključevskij (1547-1917) », Cahiers du monde russe [En ligne], 55/3-4 | 2014, mis en ligne le 08 avril 2015, Consulté le 25 septembre 2020. URL : http://journals.openedition.org/monderusse/ 8012 ; DOI : https://doi.org/10.4000/monderusse.8012 


\title{
Pierre Gonneau, Ecatherina RAI, éds. Écrire et réécrire l'histoire russe d'Ivan le Terrible à Vasilij Ključevskij (1547-1917)
}

\author{
KORINE AMACHER
}

\section{RÉFÉRENCE}

Pierre GONNEAU, Ecatherina RAI, éds. Écrire et réécrire l'histoire russe d'Ivan le Terrible à Vasilij Ključevskij (1547-1917)Paris : Institut d'Études slaves, 2013, 286 p.

1 La Russie contemporaine réécrit depuis vingt-cinq ans déjà des pans entiers de son histoire. Alors que de nombreux chercheurs analysent les réécritures actuelles de l'histoire de la Russie, le recueil collectif édité par Pierre Gonneau et Ecatherina Rai a le mérite de s'intéresser à l'écriture (et la réécriture) de l'histoire à une période plus éloignée, $\mathrm{du} \mathrm{xvI}^{\mathrm{e}}$ siècle à la veille de la révolution. Cette période historique suscite moins l'attention des chercheurs, or elle est tout aussi passionnante et instructive que la période contemporaine.

2 L'ouvrage est divisé en deux parties. La première, "Constructions et écoles historiques ", retrace l'émergence et le développement de l'histoire russe depuis le Livre des degrés de la généalogie impériale, rédigé entre 1555 et 1563, jusqu'au Cours d'histoire russe publié par Vasilij Ključevskij entre 1904 et 1911. La seconde partie, «Dramatisation et moralisation. Héros et mises en scène de l'histoire», analyse la façon dont l'histoire russe a été diversement intériorisée, mise en récit (et en scène) et interprétée.

3 La première section de la première partie, intitulée " Antiquité, sainteté et eschatologie aux sources de l'histoire russe ", s'ouvre sur une étude de Gail Lenhoff consacrée au Livre des degrés. Composée sous Ivan le Terrible, comportant dix-sept «degrés » ou 
chapitres, cette œuvre fut la première tentative de présenter l'histoire comme une succession de règnes (de Vladimir à Ivan le Terrible). L'auteur de l'article analyse plus précisément les pages consacrées à la biographie de Fedor Rostislavič, prince de la maison de Smolensk. Il montre que les compilateurs du Livre ont fait de Fedor, qui collabora avec les Mongols, un modèle d'orthodoxie, mais également un proche de Daniil, le fils cadet d'Aleksandr Nevskij et prince de Moscou auquel Fedor s'était pourtant en réalité opposé. Un soin particulier est apporté dans le Livre à dépeindre les bonnes relations entre Russes et Mongols, ainsi que le respect du khan envers l'orthodoxie. Comme le rappelle l'auteur, le Livre est rédigé alors que le khanat de Kazan' vient d'être conquis. Les relations de subordination ont changé, et la biographie de Fedor épouse dès lors les réalités politiques de l'époque. On reste dans les sources du règne d'Ivan le Terrible avec l'article d'Eugène Priadko, qui questionne la valeur historiographique du Domostroj (v. 1560), largement utilisé comme source historique par les historiens russes après sa découverte au milieu du XIX ${ }^{e}$ siècle. Dès 1872, deux philologues (I.S. Nekrasov et A.S. Mihajlov) soumettent le texte du Domostroj à une première analyse critique. Malgré son caractère polémique, l'auteur estime que la discussion entre les deux philologues a eu le mérite de démontrer l'importance primordiale d'une véritable critique textuelle pour pouvoir définir la valeur historiographique du Domostroj. L'auteur plaide lui-même pour une approche linguistique du Domostroj. Vera Tchentsova analyse comment certaines prédictions sur la chute de Constantinople ont été utilisées au milieu du XVII siècle au profit de la dynastie des Romanov par l'intermédiaire de polygraphes comme Nicolas Milescu, dit le Spathaire. Comme le montre Endre Sashalmi, après 1660, le renforcement de la légitimité de la dynastie des Romanov est clairement perceptible dans les textes et la peinture. Son analyse de l'icône peinte par Simon Ušakov en 1668 (l'Arbre de l'État russe) et de la chronique de Fedor Griboedov (Histoire des tsars et princes du pays russe, 1669) lui permet d'affirmer que la notion byzantine de "symphonie » du pouvoir cède la place à une vision qui met en avant la subordination du pouvoir spirituel au pouvoir temporel. Le Raskol vient d'avoir lieu (1666-1667) et le contexte politique a évolué. La «symphonie » du pouvoir, au cœur du Livre des degrés, est abandonnée au profit de la domination du tsar sur l'Église. Enfin, Mihail Bojcov revient sur une constante de la

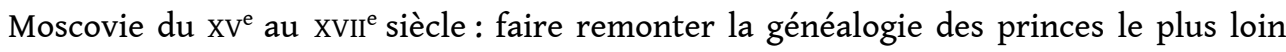
possible, pour renforcer la légitimité de la dynastie moscovite. L'auteur émet au passage des doutes sur l'importance de la théorie de « Moscou Troisième Rome » à cette période. D'autres œuvres ont eu plus d'influence, tel le Dit des princes de Vladimir, dans lequel les princes de Moscou sont présentés comme les descendants de l'Empereur Auguste, ou encore la Charte d'Alexandre, prétendument accordée par Alexandre le Grand aux Slaves. Le succès de ce faux, dénoncé par Juraj Križanič au XvII e siècle déjà, montre que «beaucoup de monde voulait croire à son authenticité ». En tous les cas, le processus de légitimation s'appuie à chaque fois sur des modèles européens, alors que d'autres traditions, en particulier orientales, auraient pu être sollicitées.

Ces cinq premiers articles témoignent que, malgré les divergences et les tâtonnements, un véritable processus de création historico-politique a lieu dans l'atelier de l'historiographe moscovite. Il s'agit de construire un "passé utile", de sacraliser le présent, de renforcer la légitimité de la dynastie des Romanov et, enfin, de justifier l'expansion de la Moscovie. Dans la section suivante («Historiennes, historiens et méthodes historiques $)$, on traverse la frontière $\mathrm{du} \mathrm{xVI}^{\mathrm{e}}$ siècle, pour évoquer la «fabrique » de l'histoire russe du XvII ${ }^{e}$ siècle à la fin du xix ${ }^{e}$ siècle. Les copistes et les 
historiographes cèdent lentement la place aux historiens «modernes » et aux écoles historiques.

5 Aleksandr Lavrov revient sur la question de l'authenticité de la Chronique de Joachim. Intégrée par Vasilij Tatiščev dans son Histoire de Russie (v. 1748-1750), elle divise les chercheurs depuis deux cents ans. Pour Aleksandr Lavrov, la Chronique a permis à Tatiščev d'asseoir son point de vue selon lequel la christianisation a été un processus conflictuel et l'Église est responsable des persécutions religieuses dans l'histoire russe. Après avoir pointé les liens entre la Chronique de Joachim et d'autres ouvrages de la même période, l'auteur estime que Tatiščev, perçu comme un "anti-normaniste ", en particulier à l'époque soviétique, voulait avant tout « produire un manuscrit ancien qui réponde à toutes les interrogations par une sorte de compromis généalogique réconciliant Scandinaves et Slaves en les mettant sur un pied d'égalité ». Enfin, il entendait également inscrire l'histoire de la Russie dans le contexte plus large de celle de l'Europe du Nord. Olga Kosheleva décrit comment l'histoire russe est lentement devenue une discipline scolaire, à partir du Synopsis imprimé à Kiev en 1674, suivi près de cent ans plus tard par la Chronique russe abrégée de Mihail Lomonosov. Il faut toutefois attendre la réforme de l'enseignement de 1786 pour que l'histoire de la Russie devienne une matière scolaire à part entière. Catherine II commande l'écriture d'un manuel d'études pour les écoles publiques. Elle a pris soin d'en définir les grandes lignes: «de la fondation de Moscou jusqu'aux Victoires, et des Victoires jusqu'à la Puissance et la Gloire ». Dans la version simplifiée qui voit le jour en 1799, les périodes de l'histoire russe sont reliées par l'idée centrale des Lumières et de la civilisation, dont les célèbres personnages de l'histoire russe sont systématiquement présentés comme les défenseurs. Mais l'histoire russe se lit aussi dans les proverbes: Stéphane Viellard consacre son article à Ivan Snegirev qui, dans une monographie en quatre livres (1831-1834), entreprit d'étudier «les Russes à travers leurs proverbes ». Il s'agit là, estime l'auteur, de la première tentative d'écrire une histoire russe dans une perspective anthropologique. La question de la méthode historique est au centre de l'article suivant. Dans quelle mesure, se demande Thomas M. Bohn, Nikolaj Karamzin, Sergej Solov'ev et Vasilij Ključevskij ont-ils adopté l'historicisme allemand en vogue au $\mathrm{XIX}^{\mathrm{e}}$ siècle? Pour l'auteur, si Karamzin et Solov'ev en ont intégré certaines caractéristiques, Ključevskij l'a dépassé et a introduit un nouveau paradigme scientifique dans l'historiographie russe: la sociologie historique. L'histoire russe n'appartient toutefois pas qu'aux historiens professionnels, comme le montre l'étude d'Angela Rustemeyer consacrée aux juristes russes de la fin du XIX ${ }^{e}$ siècle. Si certains juristes enseignent l'histoire du droit dans le cadre de la jurisprudence, les spécialistes du «droit de police» (au sens de «policer» la cité) font une large part à l'histoire. L'auteure montre que malgré leur zèle mis à défendre les droits des individus, les juristes-historiens constatent que celui-ci est avant tout la science du contrôle de l'État sur les populations, en particulier à la fin de l'Ancien Régime russe, lorsque terrorisme et lois d'exception règnent. Enfin, ils estiment tous que la « bonne police » a une origine européenne ou russe, jamais asiatique. Des juristes, on passe aux historiennes, grandes oubliées de l'historiographie russe : comme l'écrit Andreas Kappeler, l'apparition des historiennes professionnelles n'a été possible qu'à partir du moment où les femmes ont eu accès aux études supérieures. Toutefois, dès le milieu du XIX ${ }^{e}$ siècle, des femmes se font connaître comme historiennes, notamment Elena Lihačeva, qui publie dans les années 1890-1901 une histoire de l'éducation des femmes en Russie, qui fait référence aujourd'hui encore. Souvent, ces historiennes sont filles, femmes ou assistantes d'un 
professeur et sont issues des couches sociales aisées et cultivées. De même qu'en Europe occidentale, ce sont elles qui fondent l'histoire des femmes en Russie. Andreas Kappeler évoque le destin de certaines d'entre elles, historiennes pionnières ou professionnelles, nées après 1870 et dont la carrière se fait dans les milieux académiques et universitaires. Les historiennes restent toutefois trop souvent confinées dans le statut de «femmes de lettres", à l'exception notable d'Aleksandra Efimenko (1848-1918), nommée professeure à l'université de Harkov' et qui devient ainsi la première femme à obtenir une chaire d'histoire en Europe orientale et centrale. Avec Frances Nethercott, on retourne dans le terrain des historiens célèbres. L'auteure montre que Ključevskij a fait recours à de nombreuses sources littéraires (Fonvizin, Puškin, Lermontov), où il a puisé un riche matériau pour analyser les transformations de la société russe et élaborer sa compréhension des processus de transformation sociale de la Russie. La fiction l'a aidé à dépasser les facteurs économiques et à mieux appréhender les ruptures et les continuités des comportements humains. Enfin, dans l'Europe occidentale de l'époque de Ključevskij, la science historique était associée à la sociologie, à l'économie et au droit. La Russie a suivi ce modèle, mais le rôle politique et social joué par la littérature, l'autorité intellectuelle et morale des écrivains ont contribué à lisser la frontière entre les historiens et les écrivains, les uns et les autres estimant qu'ils avaient un rôle social à jouer dans un pays où le débat politique était illicite.

6 La première section de la seconde partie de l'ouvrage est consacrée aux «grandes figures du passé russe ». Sean Griffin analyse la geste de la princesse Olga dans le Récit des temps passés, ce qui lui permet d'affirmer qu'avant d'être une figure historique, Olga est une création liturgique. Sean Griffin relit le récit du baptême de la princesse olga dans le Récit à la lumière de trois livres liturgiques orthodoxes, le Typicon, le Ménologe et l'Euchologe, ce qui lui permet d'en repérer les nombreux emprunts et adaptations. Ainsi, l'auteur nous rappelle lui aussi l'importance de soumettre tout texte ancien à une solide analyse textologique avant de l'utiliser comme source historique. Florent Mouchard s'arrête sur la façon dont plusieurs chroniques moscovites, antérieures au Livre des degrés, dépeignent Vitovt (1392-1430), grand-duc de Lituanie. Si, entre 1472 et 1544 , on observe au fil des récits une évolution de l'image de Vitovt, ce dernier demeure l'archétype du " héros négatif » : rusé, menteur, vantard et orgueilleux, mais surtout dangereux pour la "vraie foi». Toutefois, Vitovt, ce «modèle réduit assez fidèle de l'attitude moscovite envers la Lituanie", n'est jamais décrit de façon totalement négative. En effet, la dynastie moscovite a des liens de parenté avec lui. Vitovt reste donc, envers et contre tout, un membre de la famille. Marcello Garzaniti montre les évolutions de l'historiographie de Maxime le Grec (v. 1470-1556). L'historiographie du XIX siècle (ainsi que soviétique) le décrit comme un adversaire de la Renaissance. Dans sa thèse, Elie Denissoff (1943), Russe émigré converti au catholicisme, nuance toutefois l'opposition farouche de Maxime le Grec au monde latin. Marcello Garzaniti plaide pour la restitution de la personnalité complexe de Maxime le Grec, qui ne privilégierait pas son combat en faveur de l'orthodoxie byzantine au détriment de l'humanisme italien, tous deux ayant influencé sa carrière et sa pensée. Pour l'auteur, cette vision, réductrice, constitue un obstacle à une étude sérieuse sur l'influence de l'humanisme en Russie. De Maxime le Grec, on passe à la figure, centrale dans l'historiographie russe, d'Ivan le Terrible. Michel Niqueux analyse la façon dont la censure théâtrale russe du XIX ${ }^{e}$ siècle a considéré le règne d'Ivan IV. Si les historiens ont eu plus de liberté pour traiter du passé (le courant représenté par le prince Ščerbatov 
et Karamzin n'est pas tendre à l'égard d'Ivan), les censeurs théâtraux sont prompts à interdire les œuvres des dramaturges dans lesquelles ils perçoivent une quelconque allusion au présent. La crainte que le public puisse y puiser des idées "séditieuses " visant à «dénigrer le pouvoir " est forte, et c'est pourquoi, même durant le règne «libéral " d'Alexandre II, la censure théâtrale reste stricte, pour des raisons tant politiques que morales et religieuses. Boris Godunov est un autre tsar qui a fait couler beaucoup d'encre. Andreas Ebbinghaus montre la construction de son image dans l'historiographie et la littérature russes. Décrit comme l'archétype du traître et le commanditaire de l'assassinat du tsarévitch Dmitrij en 1591, Boris Godunov est partiellement réhabilité par Karamzin, qui l'innocente de nombreux crimes et pointe les aspects modernisateurs de son règne. En 1829, Mihail Pogodin est le premier à montrer que Godunov n'est pas l'assassin du tsarévitch. Toutefois, au même moment, Puškin écrit son célèbre drame, qui ancre l'image d'un Godunov coupable. Enfin, chez Puškin, Boris Godunov acquiert des traits tant d'Ivan le Terrible que de Pierre le Grand, il est à la fois l'héritier du monarque médiéval et le précurseur du monarque des Temps modernes. Et plus encore que chez Karamzin, Puškin présente Boris Godunov comme un "pionnier de l'occidentalisation». Des tsars controversés, on passe aux célèbres révoltés russes. Ecatherina Rai étudie l'Histoire abrégée de Sten'ka Razin écrite par le poète et dramaturge Aleksandr Sumarokov, au moment où la révolte de Pugačev sème la terreur parmi la noblesse. Évoquant «l'amateurisme» et la «subjectivité » de Sumarokov historien, Ecatherina Rai retrace le tableau de la révolte de Razin brossé par Sumarokov. Pour lui, la révolte est un tourbillon de barbarie et la répression est justifiée. Les révoltes de Razin et de Pugačev représentent une sortie radicale des Lumières, que Sumarokov perçoit moins comme fondées sur la loi que dans le respect de la hiérarchie sociale. Enfin Gérard Laudin montre que l'histoire russe s'écrit aussi hors de l'Empire. Les Allemands (en particulier ceux qui ont servi en Russie) se sont intéressés à l'histoire russe, notamment au Faux Dimitri, ainsi qu'aux réformes de Pierre le Grand et à ses rapports avec les strel'ci. Dans la plupart de leurs drames (y compris le drame inachevé de Schiller sur Dimitri), les auteurs défendent l'absolutisme éclairé et les réformes, et questionnent les résistances que ces dernières suscitent. En arrière-fond de ces écrits, on perçoit une réflexion politique sur l'Allemagne de cette époque.

7 Les deux derniers articles de l'ouvrage sont consacrés au " pays russe et ses confins ». Comme le montre Pierre Gonneau, la mémoire collective russe cultive le souvenir de sièges héroïques, et les récits russes anciens mettent en avant la capacité de résistance $\mathrm{du}$ peuple russe. $\mathrm{Si}$, avant l'invasion mongole, le siège est décrit comme une épreuve initiatique dont l'issue est favorable aux Rus', pendant l'invasion mongole, l'esprit de sacrifice et de résistance demeure, mais la seule issue est désormais le martyre. Dès Ivan le Terrible, même si la Russie est conquérante, une mentalité de siège émerge. Les ennemis (allogènes ou intérieurs) ne peuvent être vaincus que par une résistance acharnée et par le sacrifice suprême: aucune reddition n'est permise. Le combat se mène dans les centres vitaux de la Russie comme aux confins du monde musulman. L'article de Pierre Gonneau se clôt sur l'évocation d'un récit du début du xviII siècle consacré au siège de l'abbaye de Solovki. Ce ne sont plus le tsar et l'Église qui représentent le salut et la foi : le martyr est celui qui résiste au pouvoir officiel. La fuite dans le désert, pour y vivre une vie édifiante, est une autre possibilité. Michael Khodarkovsky retrace la naissance des mythes fondateurs de l'Empire russe et les raisons de leur persistance. L'écrasante majorité des intellectuels russes $\mathrm{du}_{\mathrm{XIX}} \mathrm{e}^{\mathrm{e}}$ siècle a 
estimé que l'expansion a été exempte de violence, à la différence des impérialismes européens. À aucun moment, sauf au début du régime soviétique, lorsque l'Empire russe est dépeint comme une "prison des peuples", la Russie ne s'est considérée comme une puissance coloniale. Enfin, l'administration russe avait une connaissance sommaire des peuples qu'elle prétendait civiliser. Dès lors, «le déni de la réalité et la persistance du mythe ont donné le jour à un hybride, fait d'une construction impériale hyperaffirmée et d'un colonialisme qui ne s'avoue pas». Ce seront finalement les historiographies régionales qui, après 1991, dénonceront violemment le colonialisme russe.

En majeure partie issu d'un colloque qui s'est tenu à Paris en mai 2012, cet ouvrage passionnant offre un large tableau, temporel et thématique, d'une histoire russe qui défile à travers son historiographie. Composé de nombreuses et courtes études, d'un index et d'un précieux glossaire des principales œuvres historiques russes manuscrites citées, il laisse néanmoins parfois le lecteur sur sa faim, les faits décrits étant abordés très succinctement dans certains articles, alors que des hypothèses et idées auraient mérité un développement plus approfondi, que les contraintes éditoriales ont peut-être empêché. Les deux éditeurs écrivent dans l'introduction de l'ouvrage qu'une «bonne histoire de Russie » doit être réécrite tous les cent ans. Ne faudrait-il pas plutôt dire que chaque génération écrit sa propre histoire, qu'elle considère comme "bonne »? Inéluctablement, celle-ci sera remplacée par une autre "bonne» histoire. Hier, on écrivait des histoires nationales, aujourd'hui, on privilégie les histoires transnationales, globales, croisées. On en appelle à l'écriture d'une histoire «à parts égales " (Romain Bertrand), qui n'oublie ni ne classe les civilisations sur une échelle de valeur, comme cela fut trop souvent le cas auparavant. Tel est le destin de l'histoire, profondément liée au contexte politique et culturel dans lequel elle baigne, dont elle s'imprègne et qui lui dicte ses lois. En ce sens, cet ouvrage est éclairant, et il devrait être placé dans les mains de tous ceux qui pensent encore qu'il y a une Histoire, et non pas des histoires.

\section{AUTEURS}

KORINE AMACHER

Université de Genève 